\title{
Ocorrência de poliomielite associada à vacina no Brasil, 1995 a 2001
}

\author{
Eduardo Souza Teixeira-Rocha, ${ }^{1}$ Eduardo Hage Carmo ${ }^{2}$ \\ e José Tavares-Neto ${ }^{1}$
}

Como citar Teixeira-Rocha ES, Carmo EH, Tavares-Neto J. Ocorrência de poliomielite associada à vacina no Brasil, 1995 a 2001. Rev Panam Salud Publica. 2005;18(1):21-4.

RESUMO Objetivo. Descrever a ocorrência de poliomielite associada ao vírus vacinal no Brasil de 1995 a 2001 e determinar o risco observado para esse evento adverso.

Métodos. Este estudo retrospectivo utilizou os dados das fichas de investigação de notificações de paralisias flácidas agudas do Ministério da Saúde do Brasil. Foram considerados como casos os indivíduos com diagnóstico de paralisia flácida aguda com isolamento de vírus vacinal nas amostras de fezes ou seqüela compatível com poliomielite até 60 dias após o início da deficiência motora. Também foram incluídos os indivíduos em qualquer faixa etária que mantiveram contato com indivíduos vacinados entre 4 e 40 dias antes do início da doença e que desenvolveram deficiência motora entre 4 e 85 dias após esse contato. $O$ risco foi calculado como a razão entre o número de casos e as respectivas doses aplicadas por ano, conforme o Programa Nacional de Imunização.

Resultados. Foram registrados 10 casos de pólio associada ao vírus vacinal no período. A média de idade foi de 4,7 meses. Quatro casos foram associados à primeira dose; quatro à segunda; e dois casos foram atribuídos a contato. Os sorotipos vacinais isolados foram o tipo 1 (dois casos); 2 (um caso); e 3 (três casos). Mais de um sorotipo foi isolado em quatro casos. Houve determinação dos sorotipos nos 10 casos. O risco observado para a poliomielite associada ao vírus vacinal durante o período foi de 1:5,11 milhões de primeiras doses e de 1:10,67 milhões para o total de doses.

Conclusão. A presença de casos paralíticos provocados pela mesma política vacinal que objetiva a erradicação constitui-se no principal dilema técnico e ético da fase pós-eliminação da poliomielite.

Palavras-chave Risco, vacina antipólio oral, Brasil.

A poliomielite associada ao vírus vacinal (vaccine-associated paralytic polio-

\footnotetext{
1 Universidade Federal da Bahia (UFBa), Faculdade de Medicina, Salvador (BA), Brasil. Enviar correspondência para Eduardo Souza Teixeira-Rocha no seguinte endereço: Rua Marechal Andréa 142, apto. 601, CEP 41820-090, Salvador, BA, Brasil. E-mail: eduardo@biossaude.com.br

2 Ministério da Saúde, Secretaria de Vigilância em Saúde, Brasília (DF), Brasil.
}

myelitis, VAPP), ou poliomielite pósvacinal, é o principal evento adverso da utilização da vacina oral com vírus atenuado, descrita desde a introdução dessa vacina (1). Caracteriza-se pelo desenvolvimento, após a vacinação, de paralisia flácida aguda (PFA) idêntica a que ocorre com o vírus selvagem, podendo também determinar seqüelas motoras definitivas (2).
O mecanismo de transformação da cepa vacinal na forma patogênica é conhecido como reversão; o vírus atenuado que sofreu mutação para uma forma capaz de provocar a doença, por sua vez, é denominado vírus reverso (3). Algum grau de reversão pode ocorrer na maioria dos indivíduos receptores de vacina, porém só uma pequena parcela é capaz de desenvolver parali- 
sia por esses vírus reversos. Essa reversão à neurovirulência foi observada em crianças vacinadas com a vacina oral, principalmente após a utilização da primeira e da segunda dose (4). $\mathrm{O}$ isolamento viral em espécimes fecais de crianças que tinham recebido a vacina injetável com vírus inativo e que receberam reforço com a vacina oral também revelou a presença de reversão viral (5).

O objetivo deste estudo foi descrever a ocorrência de VAPP no Brasil no período de 1995 a 2001, assim como determinar o risco observado para esse evento adverso.

\section{MATERIAIS E MÉTODOS}

Este estudo retrospectivo foi realizado com os dados extraídos das fichas de investigação de notificações de paralisias flácidas agudas fornecidos pelo Ministério da Saúde do Brasil. ${ }^{3}$ Foram incluídos os casos oficialmente divulgados com diagnóstico de poliomielite associada à vacina ocorridos entre os anos de 1995, primeiro ano pós-certificação da eliminação da poliomielite, e 2001. Os casos foram analisados segundo o ano de notificação, a unidade federada, o sexo, a idade, a dose administrada e o vírus vacinal isolado. A medida de tendência central utilizada para a idade foi a mediana. Para os demais dados, utilizou-se a média.

Os casos foram definidos segundo os critérios do Guia de Vigilância Epidemiológica do Ministério da Saúde (6). Foram considerados como casos de VAPP os indivíduos menores de 15 anos com diagnóstico de PFA ou seqüela compatível com poliomielite até 60 dias após o início da deficiência motora, com isolamento de vírus vacinal nas amostras de fezes. As amostras de fezes não eram necessariamente oportunas (coleta nos primeiros 15 dias),

3 O Ministério da Saúde forneceu oficialmente os dados em junho de 2003. Em 8 de abril de 2005, o Ministério revisou os dados e divulgou as seguintes alterações: o número de casos se reduziu de 12 para 10. Foram excluídos quatro casos e incluídos dois. Houve identificação genética viral em todos os casos. Os dados deste artigo refletem a revisão. porém deveriam ter sido coletadas com a maior brevidade possível em relação ao início dos sintomas (6). Também foram considerados para inclusão na série os indivíduos em qualquer faixa etária que mantiveram contato com indivíduos vacinados entre 4 e 40 dias antes do início da doença e que desenvolveram deficiência motora entre 4 e 85 dias após esse contato. Quanto à análise genética dos vírus vacinais, se estabeleceu distinção entre os casos associados à vacina e aqueles denominados derivados, cuja diferença é feita pela divergência em relação ao vírus vacinal $(<1 \%$ e de 1 a $15 \%$, respectivamente).

O cálculo do risco observado foi efetuado pela razão entre o número de casos e as respectivas doses aplicadas por ano, utilizando-se os dados do Programa Nacional de Imunização (PNI) do Ministério da Saúde do Brasil, disponibilizados na Internet pelo DataSUS (7). Não foram calculados os intervalos de confiança devido à utilização de dados populacionais.

\section{RESULTADOS}

Foram registrados 10 casos de VAPP no período de 1995 a 2001. A média de idade foi de 4,7 meses, sendo todos os casos menores de 1 ano de idade (tabela 1).

O risco observado no período de estudo foi de 1:5,11 milhões de primeiras doses e de 1:3,84 milhões de segundas doses. Considerando-se todas as doses no período de 1995 a 2001 (incluídos os casos de contatos), o risco observado foi de 1 caso para cada 10667274 aplicações de doses da vacina (tabela 2).

Não ocorreram casos de poliomielite por vírus derivado do vacinal na série observada.

\section{DISCUSSÃO}

A melhor estratégia vacinal a ser utilizada para a poliomielite passou a ser questionada mais intensamente no final da década de 1980, após a cessação da transmissão do vírus selvagem em muitos países do mundo. Uma vez convivendo com a inexistência de casos provocados por vírus selvagem, os riscos da utilização da vacina oral de vírus vivos atenuados passaram a ser considerados com maior relevância, assim como as táticas para interromper a vacinação $(8,9)$.

Na série estudada, a distribuição por idade concorda com dados de estudos anteriores (4). O sorotipo menos isolado em outras séries também foi o tipo 1, sugerindo uma possível menor capacidade de reversão para a neurovirulência (10). Apenas dois dos casos foram em indivíduos que não haviam recebido a vacina (contatos), o que também está de acordo com estudos anteriores (9). Entretanto, há relatos de maior freqüência entre os contatos (11). Analisando-se a proporção por idade, observamos que o risco de desenvolver VAPP foi semelhante ao estimado em estudos anteriores (9).

Tabela 1. Casos de poliomielite associada à vacina oral, Brasil, 1995 a 2001

\begin{tabular}{lccccc}
\hline Ano & Unidade federada & $\begin{array}{c}\text { Idade } \\
\text { (meses) }\end{array}$ & Sexo & Dose & $\begin{array}{c}\text { Vírus vacinal } \\
\text { isolado }\end{array}$ \\
\hline 1995 & Rio de Janeiro & 3 & $\mathrm{~F}$ & Segunda & P2, P3 \\
1995 & Rio Grande do Sul & 4 & $\mathrm{~F}$ & Contato & P2, P3 \\
1995 & São Paulo & 7 & $\mathrm{M}$ & Primeira & P3 \\
1996 & Espírito Santo & 4 & $\mathrm{~F}$ & Segunda & P1, P2 \\
1996 & Pernambuco & 5 & $\mathrm{M}$ & Segunda & P1, P2, P3 \\
1997 & Alagoas & 6 & $\mathrm{~F}$ & Primeira & P1 \\
1999 & Piauí & 8 & $\mathrm{~F}$ & Contato & P3 \\
1999 & Goiás & 4 & $\mathrm{M}$ & Segunda & P3 \\
1999 & Bahia & 3 & $\mathrm{~F}$ & Primeira & P1 \\
2001 & Minas Gerais & 3 & $\mathrm{M}$ & Primeira & P2 \\
\hline
\end{tabular}


Tabela 2. Número de doses de vacina oral contra a poliomielite aplicadas entre 1995 e 2001 e risco de desenvolver a doença associado à primeira e à segunda dose, Brasil

\begin{tabular}{lccc}
\hline & & \multicolumn{2}{c}{ Risco $^{a}$} \\
\cline { 3 - 4 } \multicolumn{1}{c}{ Ano } & Total de doses & Primeira dose & Segunda dose \\
\hline 1995 & 12081764 & 3112276 & 2734780 \\
1996 & 10845202 & Nenhum caso & 1199626 \\
1997 & 13265702 & 3637174 & Nenhum caso \\
1998 & 16149080 & Nenhum caso & Nenhum caso \\
1999 & 18257690 & 4453513 & 3853986 \\
2000 & 18959434 & Nenhum caso & Nenhum caso \\
2001 & 17113867 & 3997649 & Nenhum caso \\
Total de doses no período & 106672739 & & \\
Risco por dose no período & $1: 10667274$ & $1: 5111921$ & $1: 3841818$ \\
Risco para total de doses & & & \\
\hline
\end{tabular}

a Risco de um caso para o número indicado de doses.

Os dados analisados evidenciam que o risco de VAPP no Brasil permanece menor do que o descrito para outras regiões ou países do mundo. Nos Estados Unidos, o risco associado à primeira dose variou de 1:750 000 (9) a 1:2,5 milhões (3) para o total de doses aplicadas. Na América Latina, esse risco foi estimado entre 1:1,5 e 1:2,2 milhões de doses aplicadas (12). No Brasil, o risco estimado para o período entre 1989 e 1995 (6) foi de 1:2,39 milhões na primeira dose e de 1:13,03 milhões no total de doses. No presente estudo, o risco associado à primeira dose da vacina se reduziu para 1 caso em 5,11 milhões de primeiras doses. Para o total de doses, o risco calculado neste estudo aumentou para 1 caso em 10,67 milhões.

A estimativa de risco no Brasil para doses subseqüentes pode sofrer influência da metodologia de dispensação simultânea da vacina oral em campanhas de vacinação e na vacinação de rotina. Nos Estados Unidos, as doses são acondicionadas em recipientes individuais e aplicadas apenas em esquemas de rotina, sendo as doses não utilizadas devolvidas ao fabricante. Isso permite um controle acurado da ordem de aplicação das vacinas e do total de doses efetivamente aplicado à população (12). No Brasil, assim como na maior parte do mundo, os recipientes que acondicionam as vacinas contêm múltiplas doses. Isso pode gerar distorções no registro da ordem de aplicação e, conseqüentemente, interferir nos resultados das análises de risco. Após 1995, com a informatização do programa de imunização, passou a ocorrer o registro da aplicação individual, personalizando os dados e tornando o sistema mais efetivo, principalmente para os dados de primeira dose.

Apesar de haver uma estratégia oficial definida pela Organização Mundial da Saúde (OMS), há importantes questões a serem definidas em relação ao melhor método para interromper a vacinação contra a poliomielite após a erradicação (13). Também ainda se estuda como devem ser as estratégias para controlar a circulação do vírus derivado da vacina oral em determinadas situações epidemiológicas de baixas coberturas. Entre as questões levantadas, a de grande relevância é ética e moral, porque a expectativa de casos de VAPP tem risco esperado e assumido pelo programa de erradicação global da poliomielite (13). Esse risco não só é inquietante, como necessita de discussão e conhecimento por parte da sociedade e dos governos que adotam essas estratégias.

Nesse contexto, há também a dificuldade técnica para manter indefinidamente a produção da vacina com vírus inativado atualmente disponível, porque a mesma compromete as estratégias de biossegurança durante $o$ processo de contenção laboratorial definitiva do vírus selvagem, estágio final da erradicação global (14). Por isso, o desenvolvimento de novas opções de modelos vacinais tem observado dois fundamentos principais: privilegiar a biossegurança, com novas formulações das atuais cepas atenuadas, e produzir vacinas com novas cepas atenuadas (15). Na atual etapa do processo de erradicação da poliomielite, é imprescindível desenvolver alternativas vacinais em caráter prioritário, sob pena de comprometer-se a meta no final do programa de erradicação global. A estratégia sugerida, mesmo com essas considerações, para controlar todos os tipos de poliomielite no período pós-erradicação, é a de progressivamente avaliar a possibilidade de substituição integral da vacina oral pela vacina com vírus inativo (13). Um estudo oficial da OMS em Cuba e outro que está em andamento na Nova Zelândia (13) vêm demonstrando preliminarmente que os vírus associados e derivados da vacina oral tendem a desaparecer depois da suspensão da mesma e de sua substituição pela vacina com vírus inativo. Entretanto, para determinar se a vacina com vírus inativo é capaz de induzir a imunidade contra o vírus derivado em regiões tropicais, estudos estão sendo desenvolvidos em Cabo Verde e na Indonésia (11).

A etapa que se segue à erradicação, até o presente momento, passa pela manutenção da vacina oral com elevados níveis de cobertura. Em conclusão, e de acordo com o planejamento estratégico da OMS, ainda será necessário conviver com casos de poliomielite associada à vacina e à circulação do vírus derivado do vacinal, por não haver melhor alternativa que o esquema atual para a proteção populacional e a efetiva interrupção da transmissão da poliomielite por vírus selvagem (16).

A ausência de circulação do vírus selvagem não é o final da história da erradicação da poliomielite. É, na verdade, o início de uma nova etapa, que exige um empenho decisivo para as ações de certificação, vigilância e contenção laboratorial e para a elaboração de políticas vacinais adequadas à nova realidade da doença (17). 


\section{REFERÊNCIAS}

1. Sabin AB. Oral poliovirus vaccine: history of its development and use and current challenge to eliminate poliomyelitis from the world. J Infect Dis. 1985;151(3):420-36.

2. Henderson DA, Witte JJ, Morris L, Langmuir AD. Paralytic disease associated with oral polio vaccines. JAMA. 1964;190:41-8.

3. Nkowane BM, Wassilak SGF, Orenstein WA. Vaccine-associated paralytic poliomyelitis. JAMA. 1987;257(10):1335-40.

4. De Oliveira LH, Struchiner CJ. Vaccineassociated paralytic poliomyelitis in Brazil, 1989-1995. Rev Panam Salud Publica. 2000; 7(4):219-24.

5. Prevots RD, Sutter RW, Strebel PM, Weibel RE, Cochi SL. Completeness of reporting for paralytic poliomyelitis, United States, 1980 through 1991. Implications for estimating the risk of vaccine-associated disease. Arch Pediatr Adolesc Med. 1994;148(5):479-85.

6. Brasil, Ministério da Saúde. Guia de vigilância epidemiológica. Brasília: Ministério da Saúde; 2003.

7. Brasil, Ministério da Saúde. DataSUS-Tecnologia da Informação a serviço do SUS. Imunizações-Doses aplicadas. Disponível em: http://tabnet.datasus.gov.br/cgi/pni/ dpnimap.htm. Acessado em 28 de setembro de 2003.
8. Centers for Disease Control and Prevention. Notice to readers: Recommended childhood immunization schedule, change in polio vaccination recommendations: sequential polio vaccination schedule, United States, 1997. MMWR Morb Mortal Wkly Rep. 1997;46(2): 35-40.

9. Prevots DR, Burr RK, Sutter RW, Murphy TV; Advisory Committee on Immunization Practices. Poliomyelitis prevention in the United States. Updated recommendations of the Advisory Committee on Immunization Practices (ACIP). MMWR Recomm Rep. 2000;49(RR-5): $1-22$.

10. Abraham R, Minor P, Dunn G, Modlin JF, Ogra PL. Shedding of virulent poliovirus revertants during immunization with oral poliovirus vaccine after prior immunization with inactivated polio vaccine. J Infect Dis. 1993;168(5):1105-9.

11. Weibel RE, Benor DE. Reporting vaccineassociated paralytic poliomyelitis: concordance between the CDC and National Vaccine Injury Compensation Program. Am J Public Health. 1996;86(5):734-7.

12. Andrus JK, Stebel PM, de Quadros CA, Olive JM. Risk of vaccine-associated poliomyelitis in Latin America, 1989-91. Bull World Health Organ. 1995;73(1):33-40.
13. World Health Organization. Global Polio Eradication Initiative-Progress 2002. Genebra: WHO, Department of Vaccines and Biologicals; 2003. (WHO/POLIO/03.02).

14. World Health Organization. Global action plan for laboratory containment of wild polioviruses. Genebra: WHO, Department of Vaccines and Biologicals; 1999. (WHO/V\&B/ 99.32).

15. World Health Organization. New polio vaccines for the post-eradication era. Geneva: WHO; 2000.

16. World Health Organization, Technical Consultative Group. Report of the interim meeting of the Technical Consultative Group on the Global Eradication of Poliomyelitis. Geneva: WHO; 2002.

17. Kew OM, Wright PF, Agol VI, Delpeyroux F, Shimizu H, Nathanson N, et al. Circulating vaccine-derived polioviruses: current state of knowledge. Bull World Health Organ. 2004; 82(1):16-23.

Manuscrito recebido em 29 de janeiro de 2004. Aceito em versão revisada em 25 de janeiro de 2005.

ABSTRACT Objective. To describe the occurrence of vaccine-associated paralytic poliomyelitis (VAPP) in Brazil between 1995 and 2001 and to determine the level of risk for this adverse event.

\section{The occurrence of vaccine- associated paralytic poliomyelitis in Brazil, 1995 to 2001}

Keywords
Methods. This retrospective study utilized data from acute flaccid paralysis investigation and notification records from the Ministry of Health in Brazil. Cases were defined as individuals with a diagnosis of acute flaccid paralysis and a positive stool culture or sequelae compatible with poliomyelitis appearing up to 60 days after the beginning of motor impairment. Also included were individuals in any age range who had contact with vaccinated persons between the 4th and 40th day before the onset of the disease and who developed motor impairment between 4 and 85 days after this contact. Risk was calculated as the ratio between the number of cases and the respective number of doses given in a year, according to the National Immunization Program.

Results. Ten cases of VAPP were recorded in the period. The mean age of the cases was 4.7 months. Four cases were associated with the first dose, four with the second dose, and two cases were attributed to contact. In two cases type 1 virus was identified; in one case, type 2; and in three cases, type 3. More than one serotype was isolated in four cases. In all 10 cases the serotype was determined. The observed risk for VAPP during the period studied was 1:5.11 million when considering only the first dose and 1:10.67 million for all the doses.

Conclusion. The main technical and ethical dilemma of the post-poliomyelitis elimination stage is the occurrence of paralytic cases that result from the vaccine policy itself, whose objective is the eradication of polio.

Risk; poliovirus vaccine, oral; Brazil. 